\title{
The Status of Deconfinement in SU(3) Gauge Theory
}

\author{
T. Çelik ${ }^{1}$, J. Engels and H. Satz \\ Fakultät für Physik, Universität Bielefeld, D-4800 Bielefeld, Federal Republic of Germany
}

Received 16 January 1984

\begin{abstract}
We assess the reliability of present lattice calculations in $S U(3)$ Yang-Mills theory at finite physical temperature. There are clear deviations from asymptotic scaling, but the ratios of deconfinement temperature, latent heat and string tension are found to remain constant under lattice changes.
\end{abstract}

\section{Introduction}

The purpose of this paper is to assess the reliability of present lattice calculations in finite temperature $S U$ (3) gauge theory, and to indicate in particular for which results a dependence on lattice size is still evident.

Before getting into details, let us note that the ultimate aim of such calculations is to provide reliable predictions for the values of temperature and baryon density at which deconfinement and chiral symmetry restoration occur - predictions sufficiently reliable to be used as input parameters for the design of heavy ion facilities and experiments. Present technical problems encountered for fermions on the lattice prevent us now from doing this for full QCD; therefore we study the pure gauge theory as a feasible test of the lattice evaluation.

The basic quantities to be calculated for the thermodynamics of $S U(N)$ gauge theory are the energy density $[1] \varepsilon$ and the deconfinement order parameter $[2,3]\langle L\rangle$; we want to study their behaviour as function of the physical temperature $T=\beta^{-1}$. On a lattice of size $N_{\sigma}^{3} \times N_{\beta}$, the energy density is given by [4]

$\varepsilon / T^{4}=6 N N_{\beta}^{4}\left[g^{-2}\left(\bar{P}_{\sigma}-\bar{P}_{\beta}\right)+c_{\sigma}^{\prime}\left(\bar{P}-\bar{P}_{\sigma}\right)+c_{\beta}^{\prime}\left(\bar{P}-\bar{P}_{\beta}\right)\right]$,

1 A.v. Humboldt fellow, on leave from Hacettepe University, Ankara, Turkey with $\bar{P}_{\sigma}$ and $\bar{P}_{\beta}$ for the space-space and space-temperature plaquette averages, respectively; $\bar{P}$ denotes the corresponding average on a large symmetric lattice $\left(\gtrsim N_{\sigma}^{4}\right)$ - it is needed to provide the correct energy density at zero temperature. On an anisotropic lattice, with spacings $a_{\sigma}$ and $a_{\beta}$, we have two couplings [5], $g_{\sigma}^{2}$ and $g_{\beta}^{2}$; for $\xi \equiv a_{\sigma} / a_{\beta}=1$, they coincide to give the usual $g^{2}$. The constants $c_{\sigma}^{\prime}=\left(\partial g_{\sigma}^{-2} / \partial \xi\right)_{\xi=1}$ and $c_{\beta}^{\prime}$ $=\left(\partial g_{\beta}^{-2} / \partial \xi\right)_{\xi=1}$ are known [6]; for the $S U(3)$ case, $c_{\sigma}^{\prime}=0.20160$ and $c_{\beta}^{\prime}=-0.13194$.

If our system at high temperature shows the behaviour of a non-interacting gluon gas, then its energy density will approach the Stefan-Boltzmann limit

$\varepsilon_{\mathrm{SB}} / T^{4}=\left(N^{2}-1\right) \pi^{2} / 15$.

On a given lattice, both high and low momenta are eliminated; the values of the energy density (1) should therefore not be compared with the continuum form (2), but rather with the energy density for an ideal gas calculated on a lattice of the same size $[7]$.

To calculate the deconfinement order parameter $\langle L\rangle$, we start with the thermal Wilson loop at a spatial site $\mathbf{x}$,

$L_{\mathbf{x}}(U) \equiv \frac{1}{N} \operatorname{Tr}\left[\prod_{\tau=1}^{N_{\beta}} U_{\mathbf{x} ; \tau, \tau+1}\right]$,

obtain the average $\bar{L}(U)$ over all sites $\mathbf{x}$ for a given configuration of $U$ 's and then average over succesive configurations. For $S U(N)$, order implies one of the $N$ physically equivalent $Z_{N}$ modes

$1, \exp \left\{\frac{2 \pi i}{N}\right\}, \exp \left\{\frac{4 \pi i}{N}\right\}, \ldots, \exp \left\{\frac{2(N-1) \pi i}{N}\right\}$.

If for any specific configuration a complex $\bar{L}(U)$ is obtained, then all link matrices for one fixed $\tau$-value 
are multiplied by the appropriate factor (4) to put the system into the sector connected to the continuum limit $U \rightarrow 1$. - The order parameter $\langle L\rangle$ indicates if the system is in a state invariant under the global center $Z_{N}$ symmetry (confinement), or if this symmetry is spontaneously broken (deconfinement) [8].

Both $\varepsilon$ and $\langle L\rangle$ are now evaluated on an $N_{\sigma}^{3}$ $\times N_{\beta}$ lattice at a given $g^{2}$. Most calculations so far are based on the Wilson action [9]

$S(U)=\frac{2 N}{g^{2}}\left\{\sum_{\text {plaquettes }}\left(1-\frac{1}{N} \operatorname{Re} \operatorname{Tr} U U U U\right)\right\}$

however, we shall also refer to some results using an alternate "improved" form [10]. Given $\mathrm{g}^{2}$, the inverse temperature $\beta=N_{\beta} a$ can only be obtained by use of the asymptotic scaling relation

$a \Lambda_{L}=\exp \left\{-\frac{24 \pi^{2}}{11 N g^{2}}-\frac{51}{121} \ln \left(\frac{11 N g^{2}}{48 \pi^{2}}\right)\right\}$

where $A_{L}$ denotes the usual lattice scale. The applicability of (6) of course has to be verified in the $g^{2}$ range studied.

The dominant feature in the thermodynamics of $S U(N)$ gauge theory, as seen on a given lattice, is the sudden increase of $\varepsilon / T^{4}$ at some coupling $g_{c}^{-2}$. Since the order parameter $\langle L\rangle$ is essentially zero for smaller and non-zero for larger $g^{-2}$, this increase is due to deconfinement. For the $S U(3)$ system, the transition is in fact clearly first order [11], and at the deconfinement point, the energy density increases by the latent heat of deconfinement.

For our assessment, we shall now proceed as follows. In Sect. II, we shall show that the critical coupling for deconfinement remains essentially constant as we vary the spatial lattice size $N_{\sigma}$ at fixed $N_{\beta}$. The dependence of $g_{c}^{-2}$ on the temporal lattice size $N_{\beta}$ at fixed $N_{\sigma}$ is studied in Sect. III; we find there that the asymptotic scaling form (6) is not yet valid for the lattices presently employed.

\section{Spatial Lattice Size}

For reference [11], we show in Fig. 1 the energy density (1), evaluated on an $8^{3} \times 3$ lattice, as function of $6 / \mathrm{g}^{2}$. At $6 / \mathrm{g}^{2}=5.55$, we have the first order deconfinement transition. The behaviour of the lattice average of the thermal Wilson loop in the deconfinement region is shown in Fig. 2, as function of the number of iterations, for each value of $6 / \mathrm{g}^{2}$ both after an ordered and a random start. While above and below $6 / g_{c}^{2}$ the system quickly reaches a single state, we observe at $6 / g_{c}^{2}$ a clear two-state signal. We

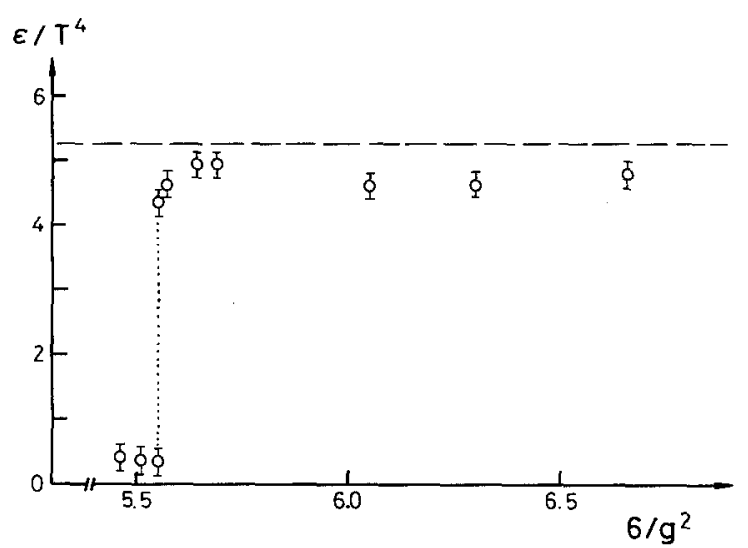

Fig. 1. Energy density $\varepsilon / T^{4}$, evaluated on an $8^{3} \times 3$ lattice, as function of $6 / \mathrm{g}^{2}$

now want to see if the value of the critical coupling depends on the spatial lattice size.

To reduce numerical work, we switch to a lattice with only two temporal lattice sites; this enhances the size of the discontinuity and reduces the number of links. We consider $N_{\sigma}^{3} \times 2$ lattices with $N_{\sigma}=6,8$, 10,12 . The resulting lattice average $\vec{L}$ is shown in Fig. 3; we note that an increase in spatial volume by a factor $2^{3}$ leaves the value of the critical coupling unaffected. We also note that the fluctuations are damped with increasing $N_{\sigma}$, reducing the risk of phase flips; below $N_{\sigma}=6$, these are so prominent that they effectively destroy the two-state signal. The value of $\langle L\rangle$ for the disordered state falls with increasing lattice size, as seen in Fig. 4; both the ordered state value of $\langle L\rangle$ and the latent heat $\Delta \varepsilon$, shown in Fig. 5, are essentially $N_{\sigma}$-independent. The constancy of $\Delta \varepsilon$ over the given range of $N_{\sigma}$ is also in accord with the constancy of the corresponding lattice results for an ideal gluon gas.

Before looking at the temporal lattice size dependence, let us briefly comment on the actual physical size of the systems so far considered. For a critical temperature $T_{c}=200 \mathrm{MeV}$, we have $a\left(g_{c}^{2}\right)=0.5 \mathrm{fm}$ on a lattice with $N_{\beta}=2$; this means spatial volumes ranging from 27 to $216 \mathrm{fm}^{3}$ as $N_{\sigma}$ varies from 6 to 12. With a $12^{3} \times 3$ lattice, with the same $T_{c}$, the spatial volume becomes $64 \mathrm{fm}^{3}$, and within a hadronic volume of size $4 \pi(0.8 \mathrm{fm})^{3} / 3 \simeq 2 \mathrm{fm}^{3}$, there are 54 lattice points. Thus both the size of the box and the mesh of the grid appear not unreasonable.

\section{Temporal Lattice Size and Scaling}

We now want to study the critical coupling as $N_{\beta}$ is varied at fixed $N_{\sigma}$. In Fig. 6, we recall the results [11] for $\bar{L}$ from $8^{3} \times N_{\beta}$ lattices with $N_{\beta}=2,3$ and 4 ; for $N_{\beta}>4$ at $N_{\sigma}=8$, phase flips again destroy the two-state signal. In Table 1 , we list the presently 

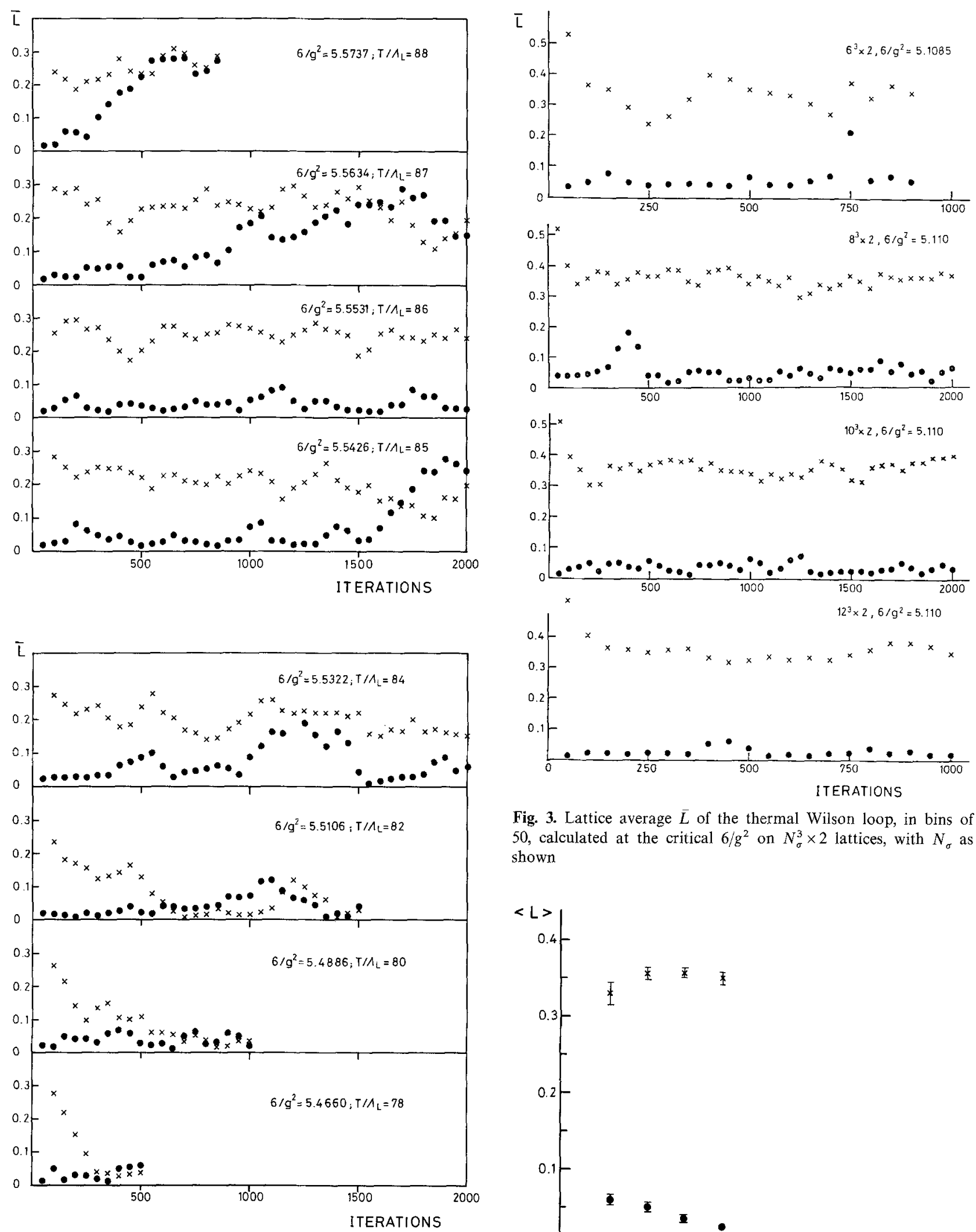

Fig. 2. Lattice average $\bar{L}$ of the Wilson loop, in bins of 50 , calculated on an $8^{3} \times 3$ lattice, as function of the number of iterations after an ordered $(x)$ and a random $(\bullet)$ start, respective$1 y$; from $[11]$

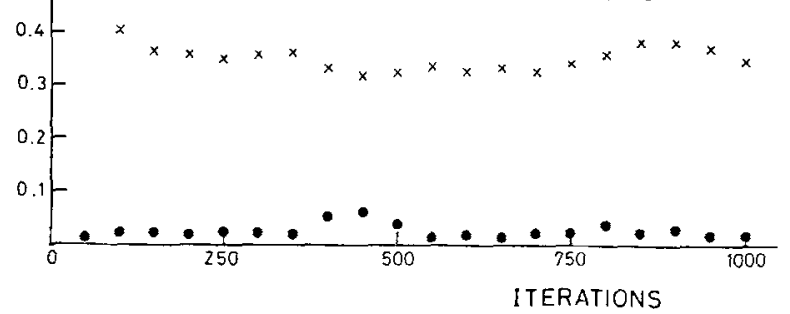

Fig. 3. Lattice average $\bar{L}$ of the thermal Wilson loop, in bins of 50 , calculated at the critical $6 / \mathrm{g}^{2}$ on $N_{\sigma}^{3} \times 2$ lattices, with $N_{\sigma}$ as shown

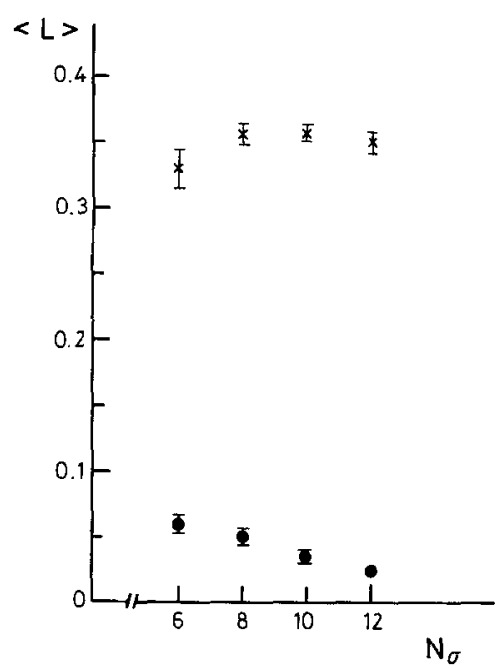

Fig. 4. Order parameter $\langle L\rangle$ at the critical $6 / g^{2}$ for $N_{\beta}=2$, as function of $N_{\sigma}$, for the ordered $(x)$ and the disordered ( $\bullet$ state 


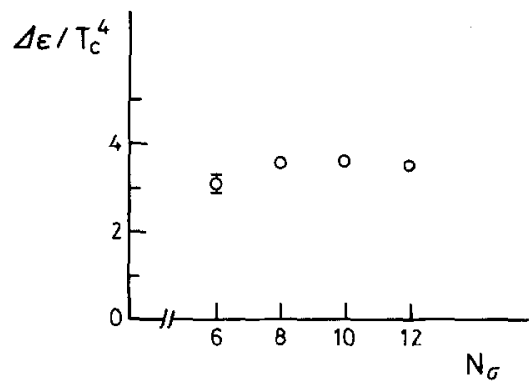

Fig. 5. Latent heat $\Delta \varepsilon / T_{c}^{4}$ calculated on $N_{\sigma}^{3} \times 2$ lattices, as function of $N_{\sigma}$

Table 1

\begin{tabular}{|c|c|c|c|c|c|}
\hline$N_{\beta}$ & $N_{\sigma}$ & $6 / g_{c}^{2}$ & $T_{\mathrm{c}} / \Lambda_{L}$ & $\Delta \varepsilon_{\mathrm{c}} / T_{c}^{4}$ & Ref. \\
\hline 2 & $8,10,12$ & 5.11 & $78 \pm 1$ & $\begin{array}{l}3.60 \\
\pm 0.10\end{array}$ & $\begin{array}{l}11 \\
\text { this paper }\end{array}$ \\
\hline 3 & 8,10 & 5.55 & $86 \pm 1$ & $\begin{array}{l}3.90 \\
\pm 0.20\end{array}$ & 11 \\
\hline 4 & 8,10 & 5.70 & $76 \pm 1$ & $\begin{array}{l}3.67 \\
\pm 0.50\end{array}$ & 11 \\
\hline 5 & 12 & $5.79-5.82$ & $68.5 \pm 1$ & & 12 \\
\hline 6 & 16 & $5.92-5.94$ & $65.5 \pm 1$ & & 12 \\
\hline
\end{tabular}

available critical coupling values, including recent results for $N_{\beta}=5$ and 6 [12]. In Fig. 7 we show the corresponding values of $T_{c} / \Lambda_{L}$ as function of $N_{\beta}$; they are obtained by use of the asymptotic scaling relation (6). Besides the just mentioned results calculated using the Wilson action, we include the analogous values obtained [13] from an "improved" action; in the latter case, $\Lambda_{L}^{\text {improved }}$ is scaled down to $\Lambda_{L}^{\text {Wilson }}$ by the observed factor 6.3 . We note that there is a clear and appearently universal systematic

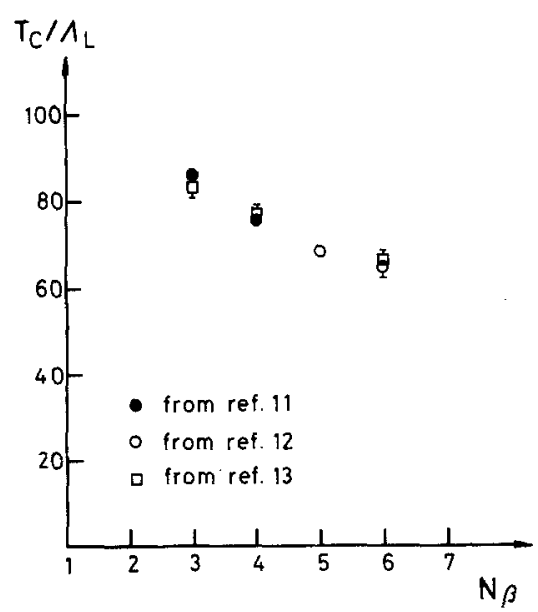

Fig. 7. Deconfinement temperature $T_{c} / A_{L}$ as function of $N_{\beta}$, for the Wilson action (circles) and the Szymanzik-improved action (squares); in the latter case, the corresponding lattice scale was converted to that of the Wilson action by use of the empirical ratio found in [13]

decrease of $T_{c} / \Lambda_{L}$ with $6 / \mathrm{g}^{2}$, indicating that in the considered range of $6 / \mathrm{g}^{2}$, relation (6) is not valid.

This could mean either that we have not yet reached the scaling regime, defined as the range of $\mathrm{g}^{2}$ where a general renormalization scheme is valid, or that only the asymptotic form (6) is not yet applicable [14]. To test which is the case we shall compare different physical quantities in the same $g^{2}$ range and see whether their ratios remain constant.

In Fig. 8, we show the dimensionless ratio $\Delta \varepsilon / T_{c}^{4}$ of latent heat and deconfinement temperature as function of $N_{\beta}$. Although $T_{d} / \Lambda_{L}$, as seen in Table 1 and Fig. 7 , is $N_{\beta}$ dependent, the ratio is constant.

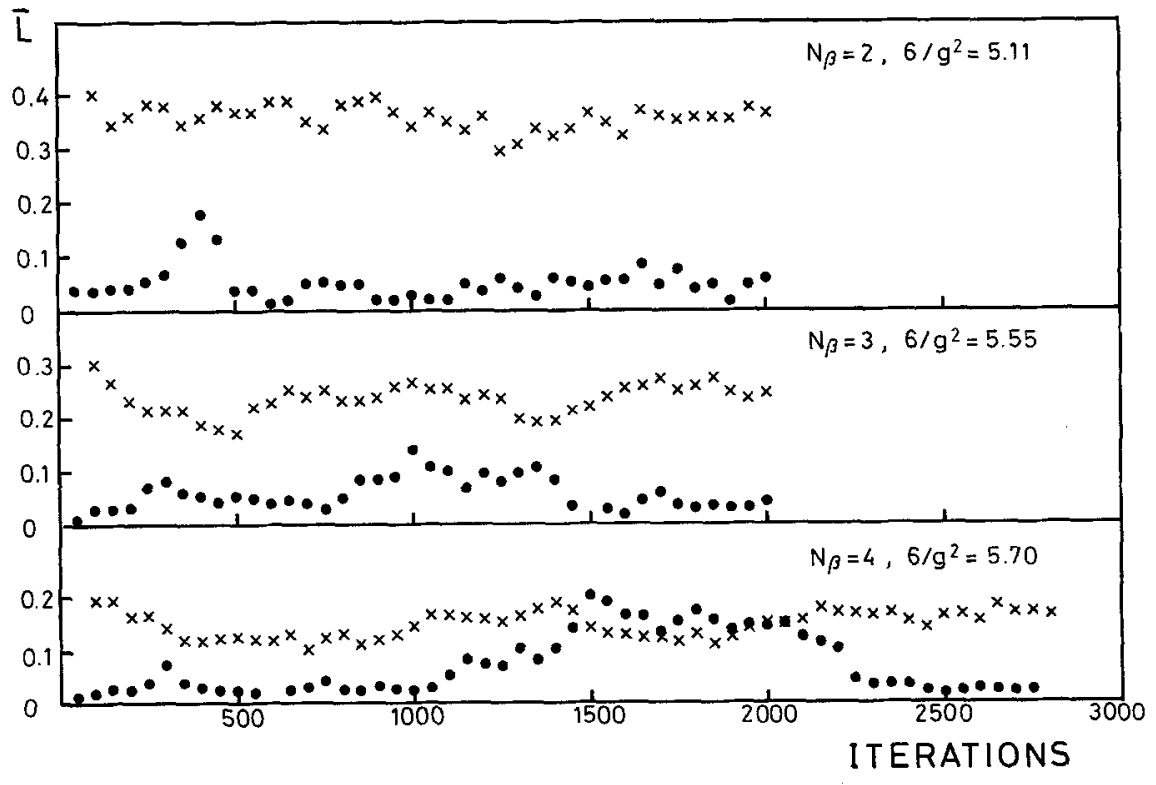

Fig. 6. Lattice average $\bar{L}$ of the order parameter, as function of the number of iterations, in bins of 50 , after ordered $(x)$ and random $(\bullet)$ starts, calculated on the following lattices: $8^{3} \times 2,10^{3} \times 3$, $10^{3} \times 4$ (crosses), $8^{3} \times 4$ (dots); from [11] 


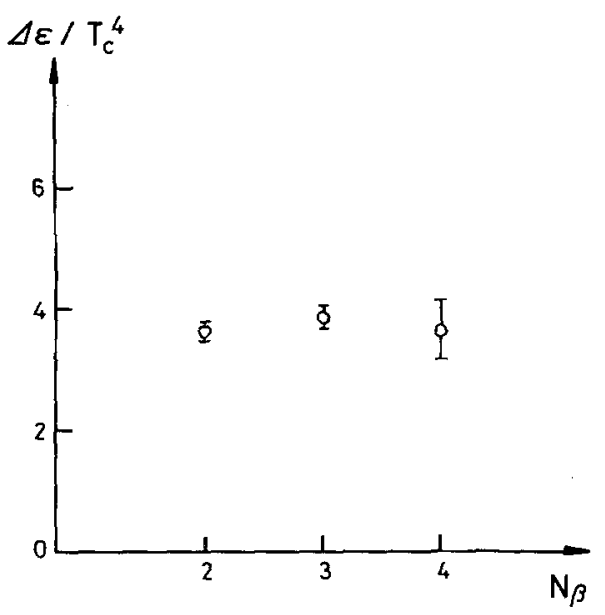

Fig. 8. Latent heat $\Delta \varepsilon / T_{c}^{4}$, calculated on $8^{3} \times N_{\beta}$ lattices, as function of $N_{\beta}$; from [11]

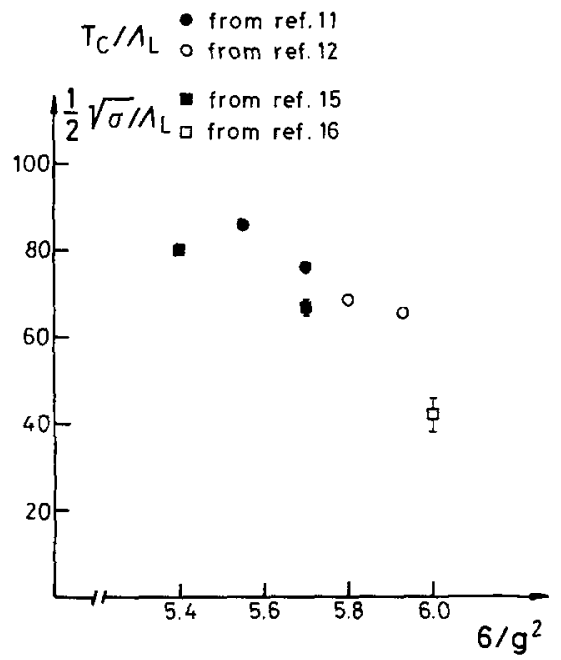

Fig. 9. Deconfinement temperature $T / A_{L}$ (circles) and string tension $\frac{1}{2} \sqrt{\sigma} / \Lambda_{L}$ (squares), as function of the coupling $6 / \mathrm{g}^{2}$

Next we combine in Fig. 9 recent results for the string tension $\sigma[15,16]$ with those for the deconfinement temperature. It is seen that both quantities exhibit the same $6 / g^{2}$ dependence in the range considered. Although the asymptotic scaling form (6) is clearly violated, the dimensionless ratio

$T_{c} / \sqrt{\sigma} \simeq 0.5$

remains independent of the choice of lattice. This result is contrary to a recent claim for an increase of $T_{\mathrm{c}}$ with lattice size [16].

\section{Conclusions}

We have seen that present deconfinement calculations are independent of spatial lattice size over a rather large range. We also find that the dimension- less ratios of physical observables $-\Delta \varepsilon / T_{c}^{4}, T_{c} / \sqrt{\sigma}-$ are independent of temporal lattice size for the range of lattices studied. Physical quantities measured in terms of the lattice scale $-T_{c} / A_{L}, \Delta \varepsilon / \Lambda_{L}^{4}$, $\sqrt{\sigma} / \Lambda_{L}-$ do, however, show a clear decrease with $N_{\beta}$ or, equivalently, with $6 / \mathrm{g}^{2}$. Hence the asymptotic scaling relation (6) is not yet valid*.

The origin of the deviation from asymptotic scaling remains unclear. The observed "physical" scaling, i.e., the lattice size independence of ratios of physical quantities, suggests that scaling in general may well be satisfied, with deviations only if we insist on a one-loop perturbative approach (6). This is in fact further supported by the similar behaviour of different lattice actions in $S U(2)$ Yang-Mills theory [14]. On the other hand, a more detailed study of the next order yields less than the observed deviation [18].

The region of couplings considered here is close to the end-point singularity in the phase diagram of a mixed fundamental-adjoint action [19], and this proximity has been considered as a possible source for scaling violations. Such an effect could be tested by varying $g^{2}$ for a mixed, rather than pure Wilson action - approaching the continuum limit along a path further away from (or closer to) the end-point singularity. The mentioned $S U(2)$ studies [14] for different actions do not, however, indicate noticeable differences in the corresponding coupling range.

It thus seems worthwhile to reach $g^{2}$ values small enough to observe at least an approach to asymptotic scaling, in the sense of a "levelling-off" of $T_{c} / \Lambda_{L}$ and $\sqrt{\sigma} / \Lambda_{L}$ in Fig. 9.

Acknowledgements. This study was intiated as preparatory work for a meeting convened in December 1983 at CERN by P. Hasenfratz. It is a pleasure to thank him and the other participants for stimulating discussions. Our calculations were carried out on the Cyber 205 of the Bochum University Computer Center, whose help is greatly appreciated.

\section{References}

1. J. Engels, F. Karsch, I. Montvay, H. Satz: Phys. Lett. 101B, 89 (1981)

2. L.D. McLerran, B. Svetitsky: Phys. Lett. 98B, 195 (1981)

3. J. Kuti, J. Polónyi, K. Szlachányi: Phys. Lett. 98B, 199 (1981)

4. J. Engels, F. Karsch, I. Montvay, H. Satz: Nucl. Phys. B205 [FS5], 545 (1982)

5. A. Hasenfratz, P. Hasenfratz: Nucl. Phys. B193, 210 (1981)

6. F. Karsch: Nucl. Phys. B205 [FS 5], 285 (1982)

* We note here, however, that calculations of the mass gap (gineball masses) so far do show scaling [17]. The cause for this discrepancy is not known; the energy density $\varepsilon$ depends only on the "local" plaquette averages and should theoretically be least sensitive to lattice size 
7. J. Engels, F. Karsch, H. Satz: Nucl. Phys. B205 [FS5], 239 (1982)

8. L.D. McLerran, B. Svetitsky: Phys. Rev. D24, 450 (1981)

9. K. Wilson: Phys. Rev. D10, 2445 (1974)

10. K. Szymanzik: Nucl. Phys. B226, 187, 205 (1983)

11. T. Çelik, J. Engels, H. Satz: Phys. Lett. 125B, 411 (1983); Phys. Lett. 129B, 323 (1983)

12. F. Karsch, R. Petronzio: Gluon thermodynamics near the continuum limit, CERN-TH 3797 (1983)

13. Ph. de Forcrand, C. Roiesnel: A study of SU(3) lattice gauge theory with next-to-nearest neighbour interactions, Ecole Polytechnique Preprint (1983)
14. R.V. Gavai, F. Karsch, H. Satz: Nucl. Phys. B220 [FS8], 223 (1983)

15. F. Gutbrod, P. Hasenfratz, Z. Kunszt, I. Montvay: Phys. Lett. 128B, 415 (1983)

16. G. Parisi, R. Petronzio, F. Rapuano: Phys. Lett. 128B, 418 (1983)

17. G. Schierholz, M. Teper: Glueball masses on large lattices. DESY-Preprint 83-107 (1983)

18. R.K. Ellis, G. Martinelli: Two loop corrections to the $A$ parameters of one plaquette actions. Frascati Preprint LNF$83 / 84$ (P) (1983)

19. G. Bhanot, M. Creutz: Phys. Rev. D24, 3212 (1981) 